\title{
Changing the Brain through Therapy for Musicians' Hand Dystonia
}

\author{
VICTOR CANDIA,${ }^{a, b}$ JAUME ROSSET-LLOBET, ${ }^{b}$ THOMAS ELBERT, ${ }^{c}$ \\ AND ALVARO PASCUAL-LEONE ${ }^{d}$ \\ ${ }^{a}$ Collegium Helveticum, ETH-Zentrum/STW, Schmelzbergstrasse 25, \\ CH-8092 Zürich, Switzerland \\ ${ }^{b}$ Institut de Fisiologia i Medicina de l'Art-Terrassa, Ctra. de Montcada 668, \\ E-08227 Terrassa, Barcelona, Spain \\ ${ }^{c}$ Department of Psychology and Lurija-Institute for Neurorehabilitation, \\ University of Konstanz, Fach D25, D-78457 Konstanz, Germany \\ ${ }^{d}$ Center for Non-invasive Brain Stimulation, Beth Israel Deaconess Medical Center, \\ Harvard Medical School, Boston, Massachusetts 02115, USA, and \\ Hospital de Rehabilitación Institut Guttmann, Barcelona, Spain
}

\begin{abstract}
Focal hand dystonia is a disorder in which sensory and motor anomalies emerge that appear to be grounded in maladaptive routes of cortical plasticity. Remodeling cortical networks through sensory-motor retuning (SMR), we achieved long-term reduction in the symptoms of focal hand dystonia. Magnetoencephalography confirmed that SMR modified the representational cortex of the fingers, whereby the representation of the affected hand was reorganized so that it resembled more the organization of the non-affected side. Furthermore, we observed differences in abnormal tactile acuity between patients with musician's cramp and those with writer's cramp: Using twopoint finger discrimination, dystonic musicians showed perceptual asymmetry between hands, while writer's cramp patients did not. To further evaluate the occurrence of collateral disturbances in focal dystonia, we assessed the clinical histories of 101 affected musicians. An important finding from this study was that dystonic musicians who play a similar first and second instrument reported a continuous worsening of their symptoms. In addition, collateral disturbances appeared with a shorter delay when more than one instrument was played. Taken together, these studies suggest that (1) neurological dysfunction can be reversed by context-specific training protocols, (2) specific symptomatic and etiological differences among various forms of focal hand dystonia might result from different behavioral experiences and their central representation, and (3) the spread of symptoms might be prevented by avoiding training that implies movement patterns similar to the main affected task, and by reducing the amount of task-associated movement behavior.
\end{abstract}

KEYWORDS: focal hand dystonia; sensory discrimination; cortical plasticity; hand rehabilitation

Address for correspondence: Dr. Victor Candia, Collegium Helveticum ETH-Zentrum/STW, Schmelzbergstrasse 25, CH-8092 Zürich, Switzerland. Voice: +41-44-632-54-04; fax: +41-44632-12-04.

candia@ collegium.ethz.ch 


\section{INTRODUCTION}

Focal hand dystonia in musicians, also termed "musician's cramp," is a greatly feared condition that leads to reduced performance levels ${ }^{1}$ and usually the termination of a musician's career. The illness is a sensorimotor disorder characterized by a loss of control over individual finger movements, especially during specific actions related to playing a given musical instrument. Hand dystonia seems to be caused or at least related to the excessive performance of repetitive activities ${ }^{2}$ and has been treated in different ways, including physical therapy, prolonged rest, anticholinergic drugs, and botulinum toxin. ${ }^{3-7}$ Nevertheless, these treatments have not induced long-term improvements ${ }^{1,8}$ even though isolated observations suggest that electromyographically guided botulinum toxin injections may have led to lasting reductions in symptoms in some of the musicians treated. ${ }^{9}$

Animal models have shown that repeated and prolonged use of the contralateral hand results in changes in the functional organization of the motor ${ }^{10}$ and the primary sensory cortex. ${ }^{11}$ Similarly, changes have been observed in the sensory cortical organization of violinists, ${ }^{12}$ and in the motor cortex of piano learners. ${ }^{13}$ Changes in auditory cortex of pianists ${ }^{14}$ and trumpeters ${ }^{15,16}$ have also been reported. It is thought that these changes underlie musical ability. However, it also seems possible to drive practice-induced changes in brain organization into the maladaptive range, a phenomenon that has been associated with focal hand dystonia. ${ }^{17-20}$ Observed sensory anomalies suggest that focal dystonia is not simply a motor problem, ${ }^{21}$ because abnormalities extend beyond the particular task affected ${ }^{22-24}$ and can be measured even during passive tactile stimulation. ${ }^{25-28}$ Abnormal sensory processing, for instance, has been repeatedly demonstrated, ${ }^{27-32}$ and it seems likely that these changes might contribute to the emergence of the disease.

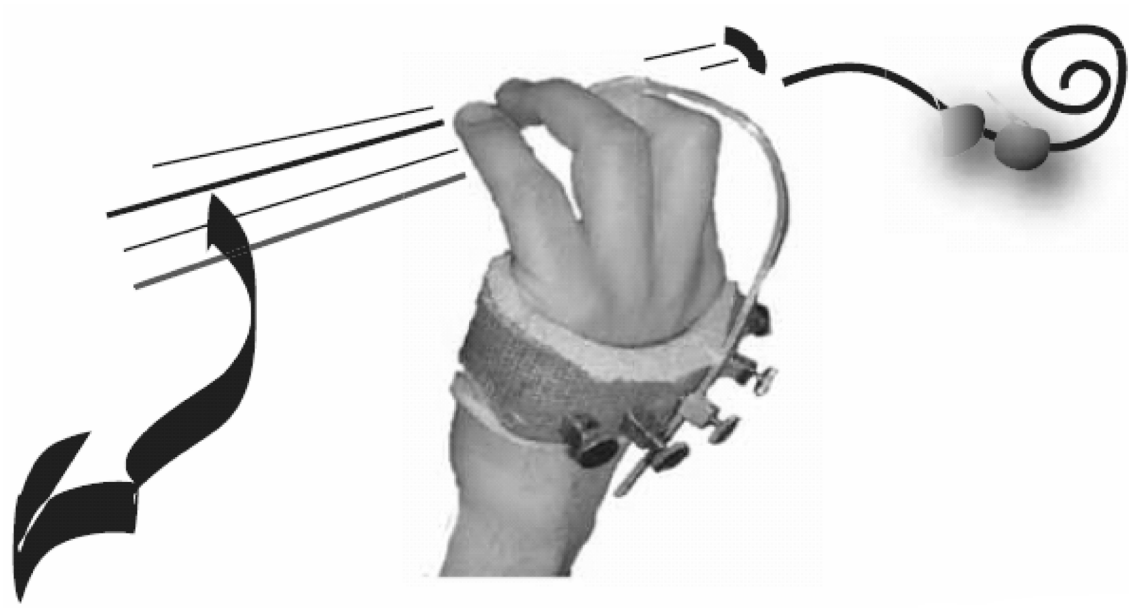

FIGURE 1. In sensory-motor retuning (SMR), a special device is used to splint fingers in such a way that dystonic movements can be avoided while practicing. It is important that the fingers can be splinted in positions that are similar to those adopted during normal playing. (Modified from Candia et al. ${ }^{35}$ ) 

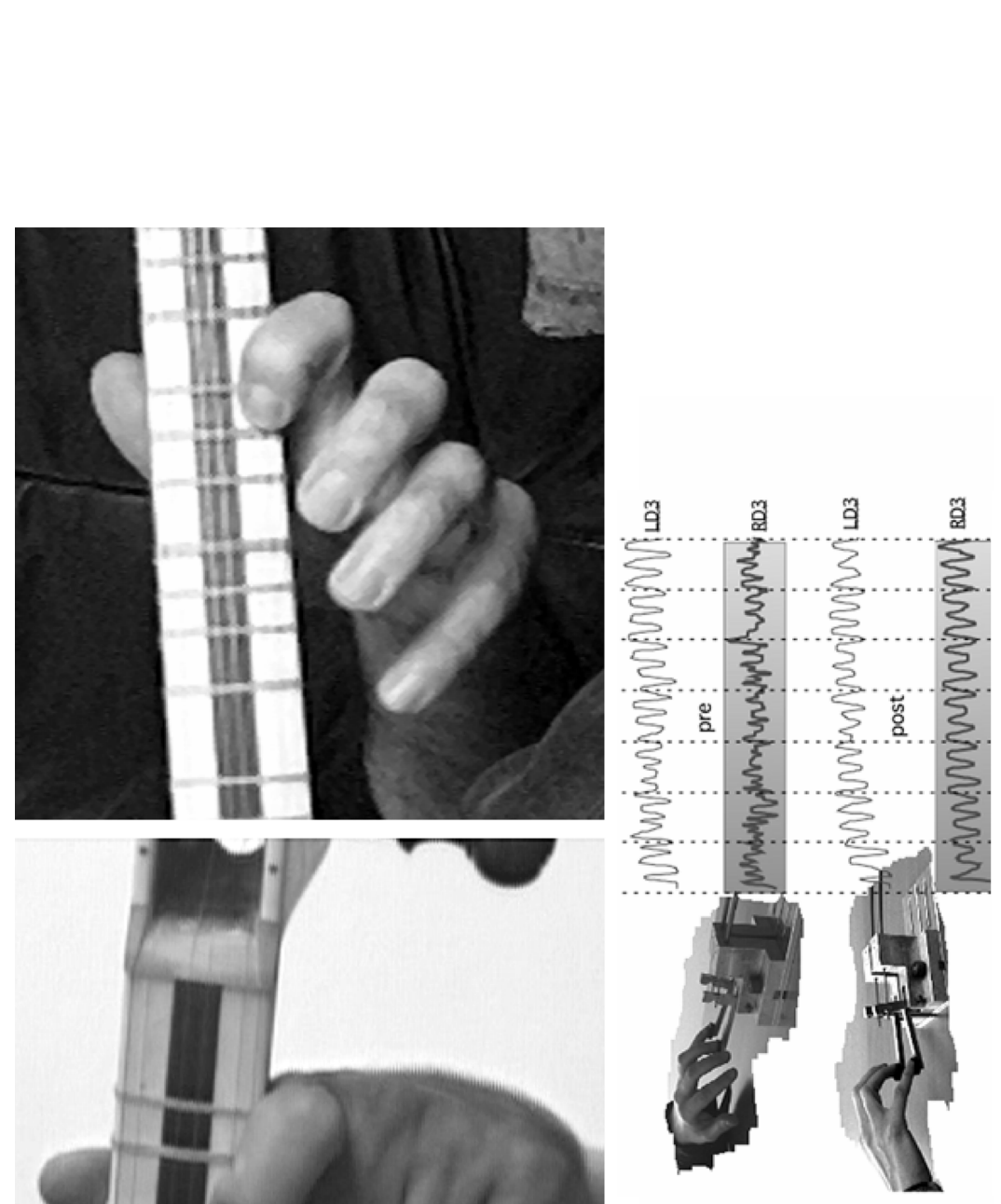

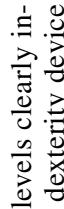

\&

ีํㄹ

훈

ญे

丞吾

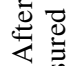

过

क च

을

車

$\Xi$

巳

넝 일

플

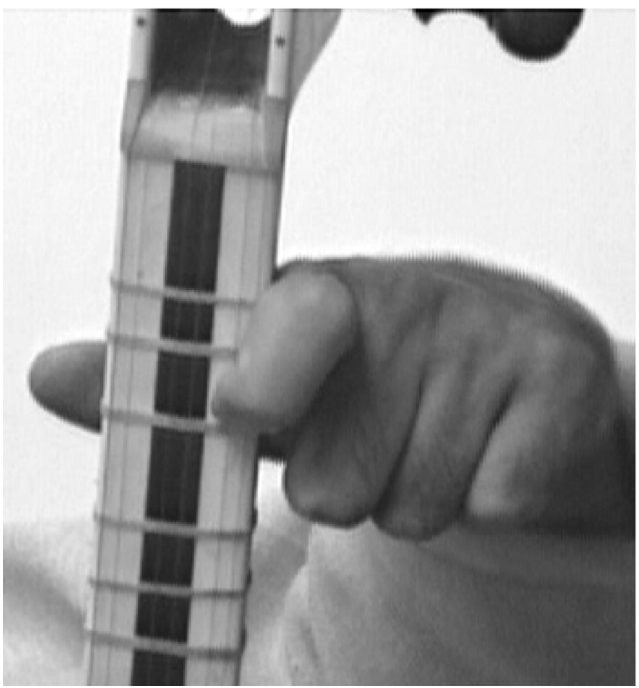

ᄋ్

콩

㭉 。

$>$

氜

3

$\Xi$ छ

옹

공

舫

징

ㅎํㅇ

광

氜

శ్
0
0
0

$\mathrm{i} \dot{\sim}$

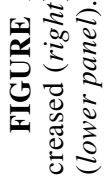




\section{RESULTS}

To evaluate the occurrence of collateral disturbances in focal dystonia, we assessed clinical histories of 101 affected musicians. ${ }^{33}$ A prominent finding was that dystonic musicians playing a similar first and second instrument (e.g., guitar and electric bass) reported continuous worsening of symptoms, whereas musicians playing only one or two rather different instruments (e.g., guitar and clarinet) did not show such a deterioration. In addition, generalized disturbances appeared with a longer delay ( $75 \%$ of cases) when only one instrument was played.

Assuming that (a) deviant brain organization contributes to focal hand dystonia and (b) motivated training can retune such brain abnormality, we developed a context-specific behavioral intervention, which we called sensory-motor retuning or SMR. ${ }^{34,35}$ All subjects treated so far presented with a chronic condition and had received diverse prior treatments for their symptoms, which, however, had resulted in little or no relief. In SMR therapy, a hand splint immobilizes one or more finger(s), allowing different permutations of finger movements on the musical instrument for short periods of time (FIG. 1).

Repertoire practice without a splint is also added in order to accomplish the transfer of the exercise-induced improvement into the real-world environment. Supervised treatment is administered for eight consecutive days for 1.5 to 2.5 hours per session, depending on the patient's fitness. ${ }^{35} \mathrm{We}$ assessed the treatment outcomes with a device that continuously measures finger displacements and a subjective dystonia evaluation scale (DES). ${ }^{36}$ Pianists and guitarists visibly improved from pre- to posttreatment (see FIG. 2). Follow-up proceeded for 3 to 25 months, suggesting that these results are long term. By contrast, the wind players did not improve. The subjective ratings and the clinical evaluation correlated with the findings obtained from the dexterity and displacement device. Thus, the movements of the dystonic fingers were smoother after SMR, indicating enhanced motor control.

To assess whether SMR would also induce observable alterations in the organization of the somatosensory cortex of the treated musicians, we studied the finger representations in somatosensory cortex in 10 patients pre- and posttreatment, using magnetoencephalography (MEG). We correlated post- and pretreatment differences between the displacement dexterity device and the patients' subjective ratings. We also correlated the difference pre- to posttreatment for the data collected with the displacement dexterity device with the difference pre- to posttreatment of the MEGrecorded dipole moment for the dystonic fingers. In addition, we calculated the cortical area of a triangle comprising the dystonic finger and its right and left neighboring fingers. These calculations showed that (1) prior to treatment, somatosensory relationships of the individual fingers differed between hands; (2) following treatment, the finger representations contralateral to the dystonic side were similar to the representation of the less affected side; (3) somatosensory finger representations were more ordered according to homuncular laws following treatment (FIG. 3); and (4) the predefined dystonic area was significantly smaller for the nontreated hand before treatment and was reduced for the dystonic hand after treatment. ${ }^{37}$ These physiological changes correlated well with the behavioral data.

To assess tactile perceptual symmetry between hands, we measured two-point finger discrimination in musicians affected in the right hand and compared the find- 

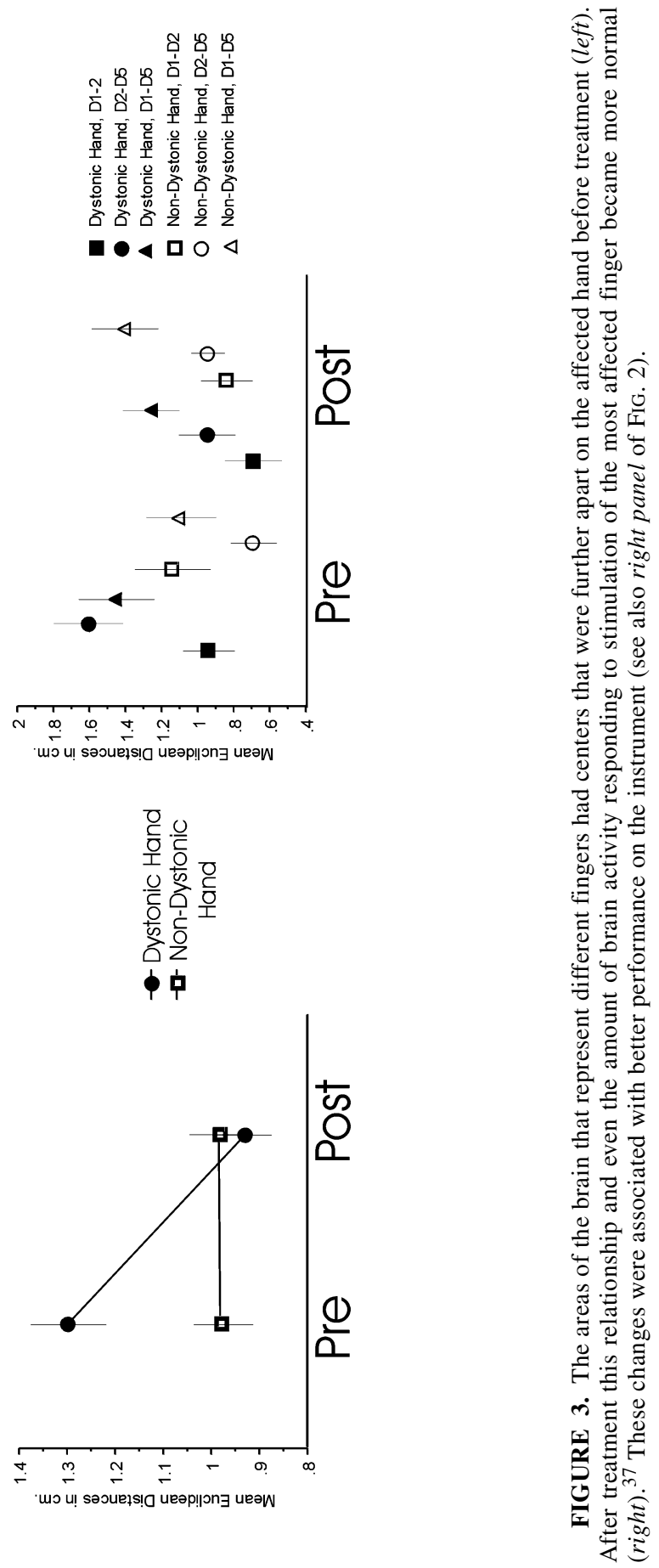


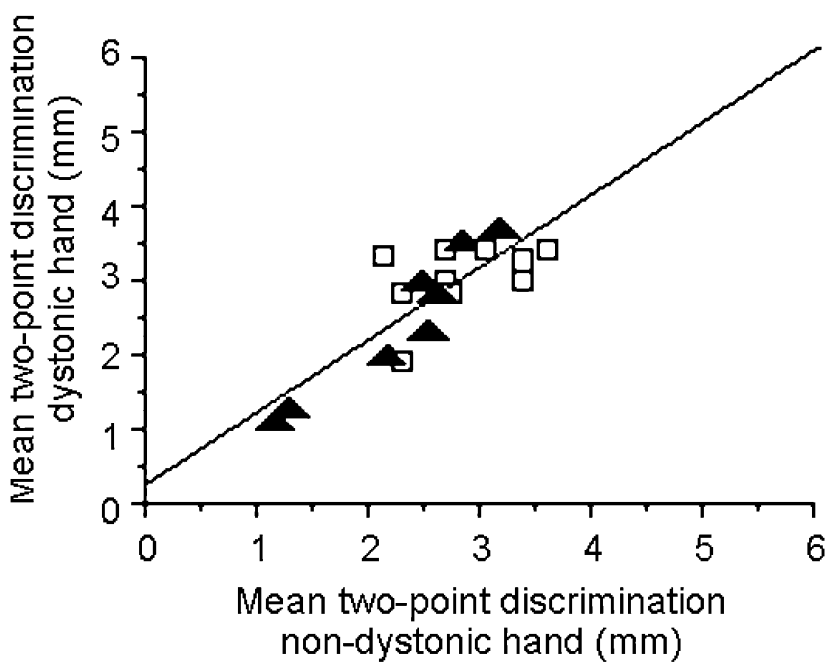

FIGURE 4. Symmetrical two-point finger discrimination was observed in the musicians' control group (filled triangles). In the dystonic group, no linear trend was evidenced (open squares). Thresholds are shown in $\mathrm{mm}$. Single values represent the average across all fingers of one hand.

ings with results in patients with writer's cramp. ${ }^{38}$ Only the dystonic musicians showed perceptual asymmetry between hands (FIG. 4).

The spatial discrimination within a defined dystonic area following the same criteria used in the MEG study revealed perceptual asymmetry within but not out of this area. Writer's cramp patients and their control group did not show such changes.

\section{DISCUSSION}

The reported data are consistent with a variety of studies that suggest that cortical organization may be modified through extensive use. ${ }^{39-41}$ Our results confirm that cortical changes, together with emergent neurological dysfunction, can be redressed by context-specific treatment. In addition, the use of a movement intervention capable of producing measurable changes in the cortical organization of sensory areas underscores the tight relationship between sensory and motor systems. This might explain the diverse anomalies reported at different levels of the sensorimotor system of the affected patients by different research groups.

It has been suggested that changes in the sensorimotor system of focal hand dystonia patients may not be exclusively limited to task-relevant stimulation but may instead be a more generalized phenomenon. ${ }^{22-24,32}$ In agreement with this notion, our data for musicians suffering from focal hand dystonia showed that changes in the symmetry of the static two-point threshold appear to be part of a more general profile of sensory anomalies: for the instruments being played by our patients, simultaneous two-point discrimination does not seem to be a task of relevance. 
Conspicuously, the assessments of the 101 affected musicians revealed that those playing similar instruments (containing similar kinematics) experience continuous aggravation of their symptoms. Moreover, those playing more than one musical instrument developed collateral disturbances faster, suggesting that increasing the degree of task similarity and the amount of practice devoted to such tasks may be crucial for worsening of symptoms. We, therefore, speculate that manual activities containing similar kinematics may lead to dystonic disturbances at some point. It is not the general reduction of muscular afferents from the affected limb ${ }^{42}$ but the specific reduction of activities containing similar kinematics that will most probably contribute to a limitation of the symptoms to a particular task, making them more amenable to context-specific interventions, such as sensory-motor retuning.

\section{ACKNOWLEDGMENTS}

Research was supported in part by the Cogito Foundation, Fundació Ciència I Art, the Deutsche Forschungsgemeinschaft, the Harvard-Thorndike General Clinical Research Center (NCRR MO1 RR01032), the National Institutes of Health (RO1MH60734, RO1EY12091, K24 RR018875), and the Goldberg Foundation.

[Competing interests: The authors declare that they have no competing financial interests.]

\section{REFERENCES}

1. Altenmüller, E. 1996. Fokale Dystonien bei Musikern: eine Herausforderung für die Musiker-Medizin. Musikphysiol. Musikermed. 3: 29-40.

2. Hallett, M. 1998. The neurophysiology of dystonia. Arch. Neurol. 55: 601-603.

3. FRY, H.J. 1993. The treatment of overuse injury syndrome. Md. State Med. J. 42: 277-282.

4. Newmark, J. \& F.H. Hochberg. 1987. Isolated painless manual incoordination in 57 musicians. J. Neurol. Neurosurg. Psychiatry 50: 291-295.

5. Lederman, R. 1988. Occupational cramp in instrumental musicians. Med. Prob. Performing Artists 3: 45-51.

6. LEVINE, W.R. 1983. Behavioral and biofeedback therapy for a functionally impaired musician: a case report. Biofeedback Self-Regul. 8: 101-107.

7. FAHN, S. \& C.D. MARSDEN. 1987. The Treatment of Dystonia. Butterworths. London.

8. BejJani, F.J., G.M. Kaye \& M. Benham. 1996. Musculoskeletal and neuromuscular conditions of instrumental musicians. Arch. Phys. Med. Rehabil. 77: 406-413.

9. Schuele, S. et al. 2005. Botulinum toxin injections in the treatment of musicians' dystonia. Neurology 64: 341-343.

10. NUDO, R.J. 2003. Functional and structural plasticity in motor cortex: implications for stroke recovery. Phys. Med. Rehabil. Clin. N. Am. 14: S57-76.

11. WANG, X. et al. 1995. Remodelling of hand representation in adult cortex determined by timing of tactile stimulation. Nature 378: 71-75.

12. Elbert, T. et al. 1995. Increased cortical representation of the fingers of the left hand in string players. Science 270: $305-307$.

13. Pascual-Leone, A. et al. 1995. Modulation of muscle responses evoked by transcranial magnetic stimulation during the acquisition of new fine motor skills. J. Neurophysiol. 74: 1037-1045.

14. PANTEV, C. et al. 1998. Increased auditory cortical representation in musicians. Nature 392: 811-814.

15. PANTEV, C. et al. 2001. Timbre-specific enhancement of auditory cortical representations in musicians. Neuroreport 12: 169-174. 
16. Pantev, C. et al. 2001. Representational cortex in musicians. Plastic alterations in response to musical practice. Ann. N. Y. Acad. Sci. 930: 300-314.

17. BYL, N.N., M.M. MERZENICH \& W.M. JENKINS. 1996. A primate genesis model of focal dystonia and repetitive strain injury. I. Learning-induced dedifferentiation of the representation of the hand in the primary somatosensory cortex in adult monkeys. Neurology 47: 508-520.

18. BYL, N.N. \& M. MelNick. 1997. The neural consequences of repetition: clinical implications of a learning hypothesis. J. Hand Ther. 10: 160-174.

19. BYL, N.N. et al. 1997. A primate model for studying focal dystonia and repetitive strain injury: effects on the primary somatosensory cortex. Phys. Ther. 77: 269-284.

20. McKenzie, A.L. et al. 2003. Somatosensory representation of the digits and clinical performance in patients with focal hand dystonia. Am. J. Phys. Med. Rehabil. 82: 737-749.

21. Pujol, J. et al. 2000. Brain cortical activation during guitar-induced hand dystonia studied by functional MRI. Neuroimage 12: 257-267.

22. TinAzZI, M. et al. 2002. Deficits of temporal discrimination in dystonia are independent from the spatial distance between the loci of tactile stimulation. Mov. Disord. 17: $333-338$.

23. Lim, V.K. et al. 2003. Perceptual differences in sequential stimuli across patients with musician's and writer's cramp. Mov. Disord. 18: 1286-1293.

24. Tinazzi, M., T. Rosso \& A. Fiaschi. 2003. Role of the somatosensory system in primary dystonia. Mov. Disord. 18: 605-622.

25. SANGER, T.D. et al. 2002. Nonlinear sensory cortex response to simultaneous tactile stimuli in writer's cramp. Mov. Disord. 17: 105-111.

26. Elbert, T. et al. 1998. Alteration of digital representations in somatosensory cortex in focal hand dystonia. Neuroreport 9: 3571-3575.

27. BARA-Jimenez, W. et al. 1998. Abnormal somatosensory homunculus in dystonia of the hand. Ann. Neurol. 44: 828-831.

28. Sanger, T.D., D. Tarsy \& A. Pascual-Leone. 2001. Abnormalities of spatial and temporal sensory discrimination in writer's cramp. Mov. Disord. 16: 94-99.

29. Bara-Jimenez, W., P. Shelton \& M. Hallett. 2000. Spatial discrimination is abnormal in focal hand dystonia. Neurology 55: 1869-1873.

30. Bara-Jimenez, W. et al. 2000. Sensory discrimination capabilities in patients with focal hand dystonia. Ann. Neurol. 47: 377-380.

31. GRUNEWALD, R.A. et al. 1997. Idiopathic focal dystonia: a disorder of muscle spindle afferent processing? Brain 120: 2179-2185.

32. Molloy, F.M. et al. 2003. Abnormalities of spatial discrimination in focal and generalized dystonia. Brain 126: 2175-2182.

33. Rosset-Llobet, J. et al. 2005. Collateral disturbances in 101 cases of musician's dystonia. Submitted for publication.

34. Elbert, T. \& B. Rockstroh. 2004. Reorganization of human cerebral cortex: the range of changes following use and injury. Neuroscientist 10: 129-141.

35. CANDIA, V. et al. 2002. Sensory motor retuning: a behavioral treatment for focal hand dystonia of pianists and guitarists. Arch. Phys. Med. Rehabil. 83: 1342-1348.

36. CANDiA, V. et al. 1999. Constraint-induced movement therapy for focal hand dystonia in musicians. Lancet 353: 42.

37. CANDiA, V. et al. 2003. Effective behavioral treatment of focal hand dystonia in musicians alters somatosensory cortical organization. Proc. Natl. Acad. Sci. USA 100: 7942-7946.

38. Candia, V., A. Pascual-Leone \& T. Elbert. 2005. Two-point discrimination in the fingers is different in patients with musician's or writer's cramp. Submitted for publication.

39. STERR, A. et al. 1998. Changed perceptions in Braille readers. Nature 391: 134-135.

40. STERR, A. et al. 1998. Perceptual correlates of changes in cortical representation of fingers in blind multifinger Braille readers. J. Neurosci. 18: 4417-4423.

41. Pascual-Leone, A. et al. 1995. The role of reading activity on the modulation of motor cortical outputs to the reading hand in Braille readers. Ann. Neurol. 38: 910-915.

42. PRIORI, A. et al. 2001. Limb immobilization for the treatment of focal occupational dystonia. Neurology 57: 405-409. 